\title{
Trayecto y situación de la ciencia política en Jalisco vistos por cuatro de sus artífices
}

\section{Route and situation of political science in Jalisco from four of its architects}

doi: http://dx.doi.org/10.32870/espiral.v26i74.7067 Alberto Arellano Ríos

\begin{abstract}
Resumen
En este artículo se reflexiona sobre el trayecto y situación de la disciplina politológica en la segunda más grande comunidad epistémica de ciencias sociales de México, ubicada en el estado de Jalisco. El ensayo se estructura en tres apartados. En el primero, se contextualizan de manera breve la travesía y discusiones que ha tenido la ciencia política en el ámbito nacional. En el segundo, se da cuenta del trayecto de los programas académicos que hay en el estado en ciencia política, tanto de pregrado como de posgrado, así como de los estudios e investigación de los temas políticos en la misma entidad. En el tercer apartado, se reflexiona la disciplina en Jalisco a partir de lo que valoran cuatro de sus artífices o actores claves de su institucionalización.

Palabras clave: ciencia política, Jalisco, reflexiones, artífices, comunidad epistémica.
\end{abstract}

\begin{abstract}
In this paper, the course and situation of the political science at Jalisco, México, is reviewed, as the second largest epistemic community of the country. The document is divided into three sections. In the first one, we briefly contextualize the course and discussions that Mexican political science has had at national level. In the second one, we demonstrate the path of academic programs of political science in the state, both undergraduate and postgraduate studies, as well as research about political issues in Jalisco. In the third section, we ponder trends in the discipline from this part of the country using the opinions of four of their architects.
\end{abstract}

Keywords: Political science, Jalisco, reflections, architects, epistemic community.

-Profesor-Investigador de El Colegio de Jalisco, México. ORCID: http://orcid.org/0000-0003I227-4087—aarellano@coljal.edu.mx

Fecha de recepción: 28 de agosto de 2018. Fecha de aceptación: 29 de octubre de 2018. 


\section{Introducción'}

¿Qué recuento puede hacerse sobre el trayecto, la situación y las expectativas de la ciencia política en el estado de Jalisco, México? Esta es la cuestión central que articula este ensayo académico. Las respuestas serían múltiples y variadas, debido a las aristas que la pregunta tiene. Antes de dar cuenta de algunas de ellas, es necesario sostener que la ciencia política en esta entidad federativa está al tanto de las grandes discusiones y los debates que acontecen en la disciplina en el resto del mundo, así como de las ciencias sociales en general. Sin embargo, en el balance que se puede hacer de al menos tres de sus elementos - su trayecto, su institucionalización y su quehacer académico vinculado-, hay asimetrías que hacen que la ciencia política del estado no se inserte finalmente, de una mejor manera, en una discusión y reflexión teórica de mayor alcance, ni aborde los grandes problemas del propio estado y del país en general, ni mucho menos los solucione. ${ }^{2}$

Se debe precisar que algunas de las reflexiones, ideas y evidencias que respaldan este texto académico descansan en estudios previos que reflexionan el estado y situación de la ciencia política en América Latina y México (Acosta Silva, 2009; Aguilar Rivera, 2009; Alarcón Olguín, 2006 y 2012; Aziz Nassif, 1999; Barrientos del Monte, 2013, 2014, 2015 y 2017; Bulcourf, Gutiérrez, y Cardoso, 2015; FloresMariscal, 2016; Loaeza, 2005; Reveles Vázquez, 2012;

I.El autor agradece las observaciones y comentarios hechos por los evaluadores anónimos. Se señala que el texto mejoró gracias a ellos. De lo contrario, se asume la responsabilidad ante toda falla o debilidad del material.

2. Para los fines del presente artículo, por institucionalización se debe entender la creación, fundación y desarrollo de programas de pregrado y posgrado en ciencia política en las instituciones de educación superior o centros de investigación. Por quehacer académico, si bien se implica la docencia e investigación en temas u objetos de la ciencia política, sólo se considera la generación y difusión del conocimiento de la comunidad científica en esta entidad federativa de México, así como su reflexión en torno a estos asuntos.

\section{0}


Rivera y Salazar-Elena, 2011; Sánchez Noriega Armengol, 2015; Suárez-Iñiguez, 2014; Vidal de la Rosa, 2013), así como en aquellos que analizan a la ciencia política hecha en Jalisco (Arellano Ríos, 2010, 2013, 2014, 2015 y 2017; Gómez Valle, 2011). En todos estos estudios, se analizan la creación de escuelas y facultades de ciencia política, su historia, el tipo de formación que se da en pregrado y posgrado, los programas educativos, sus debates internos, su relación con otras disciplinas, etc., en distintos lugares del país.

El presente artículo, por su parte, pretende sumarse a dichos estudios. Para ello, hace además algo hasta ahora poco ejecutado: se entrevista a actores que con el paso del tiempo han sido reconocidos por su papel central en los procesos de institucionalización académica de la ciencia política en el occidente de México.

Entonces, para valorar no sólo el trayecto sino la situación de la disciplina a partir de lo que se percibe sobre ella desde Jalisco, este texto se vale de cuatro entrevistas hechas a académicos e investigadores que son artífices, directos e indirectos, en el proceso de instauración de la ciencia política en esta región del país.

En este punto, vale la pena precisar dos cuestiones. Por un lado, más que ver en el texto un provincianismo o parroquialismo, se invita a observar cómo desde un territorio específico se hace presente la multidimensionalidad de la realidad o, en otra palabras, cómo actores e instituciones locales y regionales se interrelacionan y debaten con otras ideas y espacios nacionales y globales. Por otro lado, se destaca que la entrevista no sólo sirve para obtener información, pues aunque se puede cuestionar su objetividad como método de obtención de datos, lo que al final hace es dar cuenta de perspectivas, posiciones o vivencias que articuladas a un contexto ayudan a comprender y encontrar 
sentido lógico y significado a los procesos políticos, sociales, económicos o culturales. ${ }^{3}$

Los cuatro entrevistados para este trabajo fueron Adrián Acosta Silva, Jaime Preciado, Javier Hurtado y Jorge Alonso. Se trata de estudiosos de temas políticos, miembros del Sistema Nacional de Investigadores (SNI) quienes, en sus diferentes perspectivas, pueden dar luces y aportar ideas a partir de su experiencia y trayectorias académicas. ${ }^{4}$

Como punto de partida, este texto asume dos supuestos con una finalidad heurística: 1) hay ciencia política en Jalisco, y sus características, fortalezas y debilidades son materia de reflexión de este documento, aunque el debate al respecto sigue abierto; y 2) los entrevistados han tenido labores académicas, pues han encabezado proyectos y pro-

3. Las entrevistas realizadas fueron semiestructuradas. Se parte de la concepción de una entrevista como "una conversación que se propone con un fin determinado distinto al simple hecho de conversar" (Díaz-Bravo, Torruco-García, MartínezHernández, y Varela-Ruiz, 2013, p. 163). La entrevista es una técnica de gran utilidad en la investigación cualitativa cuya intención inicialmente es recabar datos, pero también sistematizar información y obtener ideas detalladas y reflexiones profundas acerca de un tema en específico (Díaz-Bravo,Torruco-García, MartínezHernández, y Varela-Ruiz, 2013).

4. Javier Hurtado fue el fundador de la Licenciatura en Estudios Políticos y Gobierno en la Universidad de Guadalajara. Jaime Preciado es el impulsor de los posgrados (maestría y doctorado) en ciencia política de la misma institución, los cuales han sido catalogados en el Programa Nacional de Posgrados de Calidad (PNPC) del Consejo Nacional de Ciencia y Tecnología (Conacyt) en la categoría de de reciente creación. Ambos fueron jefes del Departamento de Estudios Políticos de la misma universidad, en diferentes periodos. Javier Hurtado es además especialista en temas de sistemas de Gobierno y elites políticas. Jaime Preciado, por su parte, es reconocido en temáticas de la geografía electoral. Por otro lado, Jorge Alonso, además de ser investigador nacional emérito del SNI, es pionero en los estudios electorales a nivel nacional. Finalmente, Adrián Acosta Silva coloca y sitúa perspectivas politológicas en el estudio de la educación superior, y también reflexiona sobre el estado de la ciencia política. Cabe aclarar que en la lista de entrevistados no se encuentra Juan Manuel Ramírez Sáiz, quien ha sido un destacado y prolífico investigador en temáticas de participación ciudadana y movimientos sociales desde Jalisco. No se trata de una omisión, sino que se experimentó dificultad para entrevistarlo debido a que atravesaba problemas de salud. 
gramas vinculados directa o indirectamente con la construcción de la ciencia política en el estado.

El artículo se estructura en tres apartados. En el primero, se contextualiza de manera breve la travesía de la ciencia política en México. En el segundo, se da cuenta del trayecto de los programas académicos en ciencia política y la investigación en temáticas políticas en Jalisco. Finalmente, el tercer apartado es de índole reflexiva, pues valora el tipo de disciplina que se debe instituir a partir de lo dicho por los cuatro entrevistados.

\section{La ciencia política mexicana: un recuento sucinto}

Como se asentó antes, el estudio y reflexión en torno a la situación y el balance de la ciencia política en México ha quedado plasmado en diversos artículos y ensayos académicos. Es posible decir que desde que Sartori (2004) cuestionó a la disciplina que se practicaba en los Estados Unidos de América, la discusión al respecto se incrementó en el mundo y, desde luego, en México.

En el marco anterior, y para hacer un balance sucinto de la situación de la ciencia política en el país, conviene rescatar el texto de Soledad Loaeza (2005) en que precisa que la disciplina en México tuvo la virtud de tomar el ritmo político del cambio al régimen. Como dicha autora señala, la ciencia política en el país tomó el pulso del camino hacia la democratización y sus problemas de congestionamiento desde la década de 1970. A partir de entonces, la disciplina en México experimentó un auge sin precedentes, con la progresiva creación de diversos centros de investigación (Loaeza, 2005, pp. 192-203).

En el contexto anterior, y haciendo una mirada rápida de la evolución disciplinar, Loaeza (2005) plantea que dicho auge se debió al agotamiento del paradigma marxista en el estudio de los fenómenos políticos del país y el surgimiento 
de la llamada ciencia política de carácter liberal. Dicho cambio de paradigmas fue más nítido desde la década de 1980, cuando la ciencia liberal se posicionó poco a poco en la academia y logró tener un lugar protagónico, lo que fortaleció a la comunidad científica y originó disputas y debates intelectuales (Loaeza, 2005).

Por su parte, en cuanto a las ideas y enfoques de trabajo de la disciplina, Adrián Acosta Silva elaboró un mapa de las orientaciones, agendas, problemas y métodos utilizados en el análisis politológico mexicano. En el primer periodo estudiado (1980 a 1990) por Acosta Silva, el investigador encontró que los temas analizados fueron los límites del autoritarismo y la crisis del régimen político, con problemas de investigación como el presidencialismo, la caracterización del régimen político, el sistema de partidos y la reforma electoral.

Luego, en el periodo 1990-2000, los temas de agenda de la ciencia política en México fueron la transición y la democratización, con problemas de investigación como las elecciones, los partidos e instituciones políticas, la gobernabilidad y el cambio político, la cultura política, los Gobiernos locales, la alternancia política, el Gobierno y las políticas públicas, así como los Gobiernos divididos.

Finalmente, en los años que van de 2000 a 2008, el tema de agenda fue principalmente la consolidación democrática, y los problemas investigados fueron la evaluación de la transición política, los Gobiernos locales, los Gobiernos divididos y la relación entre poderes, las políticas públicas, la gobernanza, así como la participación, la democracia y el desarrollo (Acosta Silva, 2009, pp. 10-128).

En cuanto a los objetos de estudio de la ciencia política en México, Javier Hurtado ha señalado que la disciplina lleva años concentrada en temáticas de acceso al poder, y no en el ejercicio y la distribución del mismo. Esta es una de sus limitaciones (entrevista personal, 2015b). Para 
el propio Hurtado, desde 1977, cuando se implementó la reforma política electoral que abrió espacios a la oposición del Partido Revolucionario Institucional (PRI), los asuntos electorales, con sus procesos y organismos, imperan en los estudios politológicos del país (entrevista personal, 2015b).

Sin duda, las elecciones federales de 1988 marcaron otro gran momento en los estudios de la ciencia política mexicana, y después, en la década de los noventa, el surgimiento del Instituto Federal Electoral (IFE) cristalizó muchas de sus propuestas. En esa misma década, la ciencia política mexicana se abocó a los estudios de los procesos de transición en la democracia a través de las elecciones, lo que hizo surgir una escuela en el mundo y en América Latina liderada principalmente por Philippe Schmitter y Guillermo O’Donnell. Aunque al final objeto de estudio de los transitólogos se desvaneció, los electorólogos siguen existiendo debido a que su objeto de estudio es más estable. Para Javier Hurtado, la temática de los estudios electorales provocó incluso que se instaurara un modus vivendi al respecto, el cual, desde el punto de vista burocrático, generó que las escuelas y las facultades de ciencia política del país se concentraran en producir y formar funcionarios e investigadores electorales (entrevista personal, 2015b).

La ciencia política en el país ha construido debates en materias vinculadas con lo electoral. Uno de ellos, abordable desde la ciencia política o desde otras disciplinas sociales, tiene que ver con el ejercicio actual del poder vía las elecciones, como señala Jorge Alonso:

[...] las elecciones ya no definen nada, y votar o no votar es lo de menos [...], quienes tienen el control no son ni siquiera los partidos ni los Gobiernos: quienes tienen el control de todo esto son las grandes corporaciones transnacionales, el capital financiero, los grandes poderes fácticos, los grandes medios electrónicos y el crimen organizado. Ahora el crimen organizado está metido en la cuestión electoral:no solamente 
manda candidatos, [sino que también] manda dinero a las campañas, que no se puede rastrear aunque se vea (entrevista personal, 20l5d).

La concentración de la disciplina en las temáticas electorales ha condicionado que la ciencia política mexicana esté lejana de la discusión sobre los procesos de distribución, ejercicio y control del poder político. Este vacío dejado al respecto por la ciencia política ha sido zanjado por el trabajo de instituciones enfocadas en otras disciplinas, como el Instituto de Investigaciones Jurídicas de la Universidad Nacional Autónoma de México (UNAM), en su caso desde estudios sobre derecho constitucional. Por ello, Javier Hurtado sostiene que, más que ciencia política en México, lo que existe es

[...] básicamente antropología política, sociología electoral o sofisticados estudios electorales de ciencia política, pero no hay una reflexión institucional sobre el fenómeno del poder [...], la configuración de los órganos de poder, la integración de los órganos de poder, de las relaciones que deben existir entre los poderes, de la distribución del poder en un sistema federal entre órdenes de Gobierno, ni sobre los mecanismos de control del poder por el poder mismo, eso no existe, eso lo estamos viendo, esa es la gran asignatura pendiente en nuestro país [...]. [Esto deviene en] los problemas de centralización que se están dando (entrevista personal, 20I5b).

Era natural que las primeras versiones de la ciencia política en México estudiaran esas cuestiones electorales o analizaran la transición, pero lo sustancial es que otras áreas de estudio propias de la ciencia política han sido olvidadas por dicha disciplina en el país. Como asienta Hurtado (entrevista personal, 2015b), la ciencia política mexicana poco o nada ha hecho sobre el estudio institucional del poder. Tampoco ha configurado estudios sobre las élites, como los 
creados por autores como Roderic Ai Camp y Peter Smith (entrevista personal, 2015b). ${ }^{5}$

Como puede notarse, el debate sobre la ciencia política en México se ha abierto e intensificado. En otra arista, Antonio Aguilar Rivera (2009) reflexionó no sólo las condiciones en las que se practicaba la ciencia política en el país, sino sobre su calidad y sus contrastes. El autor planteó que en México había archipiélagos y enclaves politológicos que ejercían la disciplina siguiendo los modelos de investigación de Estados Unidos, los que a su vez consideraban las producciones académicas del país como de segunda o tercera categoría. Aguilar Rivera (2009) también observó que el rasgo central de la ciencia política mexicana es su fragmentación, además de ser plural teórica- y metodológicamente, endogámica y autorreferencial. Ante los graves problemas que aquejan al país, el mismo autor ha cuestionado severamente que la ciencia política mexicana no produzca conocimiento significativo a partir de preguntas realmente importantes (Aguilar Rivera, 2009).

Ante estos planteamientos, Mauricio Rivera y Rodrigo Salazar-Elena (2011) se propusieron desmentir la idea de que la ciencia política mexicana estaba siguiendo el modelo estadounidense. Los autores propusieron dejar el terreno de las impresiones y evaluaron doscientos treinta y un trabajos publicados en tres revistas académicas mexicanas durante

5. Javier Hurtado sostiene que los estudios de las élites políticas mexicanas están estancados "porque paradójicamente ahora que estamos en la época de la transparencia y del acceso a la información es cuando menos existe información sobre las élites políticas, simplemente porque ahora [esa] es información reservada ○ [considerada] información personal. Era más fácil en el Estado autoritario mexicano conseguir información sobre las élites políticas que hoy, en el Estado supuestamente democrático... Eso ha venido a [generar] que se apaguen los estudios de las élites políticas, porque es muy difícil encontrar información sobre las [mismas],y entonces [...] todo el auge en la disciplina lo va teniendo la disciplina de los estudios electorales" (entrevista personal, 2015b). 
el periodo 1990-2007. ${ }^{6}$ A partir de dicha muestra, los autores sostuvieron enfáticos que la ciencia política mexicana es predominantemente descriptiva, monográfica, cualitativa, centrada en los estudios de caso y generadora de hipótesis por la vía inductiva. Notaron también que había una alta similitud en términos de agenda de investigación entre las publicaciones políticas mexicanas y las estadounidenses, y concluyeron que debatir en la disciplina la preeminencia de algún método en particular contribuiría más a la misma en la medida en que se discutieran aspectos sustantivos a los procesos mismos de investigación.

En cuanto a los problemas profesionales e institucionales que tiene la ciencia política mexicana, Godofredo Vidal de la Rosa (2013) sostuvo que el principal de ellos es su desorganización interna. También destacó el hecho de que su profesionalización no necesariamente implicara su maduración como disciplina, o bien, que a pesar de poseer ya un aura de expertos en ciertos temas no se estuviera dando un debate teórico-metodológico profundo. Para Vidal, esto sucede porque el problema nodal de la disciplina, no sólo en México sino en el mundo, es de índole teórica, y evade el problema de la democracia (Vidal de la Rosa, 2013, pp. 89,98 y 103).

Por su parte, Enrique Suárez-Iñiguez (2014) identificó como problemas de la ciencia política en México: 1) su división interna; 2) que la disciplina aún está atada a la administración pública; 3) que no amplía sus perspectivas geográficas, pues sólo trabaja temas o problemas exclusivamente de México; 4) que no abre el espectro de los problemas políticos de estudio; y 5) que no aumenta el número de editoriales y revistas que publican trabajos de la disciplina (Suárez-Iñiguez, 2014, pp. 178-183).

6. Las revistas evaluadas fueron Política y gobierno, del Centro de Investigaciones y Docencia Económicas (CIDE), Revista mexicana de sociología, de la UNAM, y Foro internacional, de El Colegio de México.

\section{8}


Otra interpretación más optimista, que asume que la ciencia política va hacia la fortaleza institucional y la madurez disciplinar, se encuentra en el trabajo de Joel FloresMariscal (2016), quien analiza la literatura politológica mexicana e identifica tres grandes periodos. El primero de ellos, una etapa inicial, fue de 1947 a la década de los setenta. Fue una etapa en que se crearon instituciones y medios de publicación académica. A esta siguió un segundo periodo, llamado de maduración, que fue de 1980 a 1990. En este segundo periodo, Flores-Mariscal nota un incremento en los títulos de medios impresos y una diversificación del perfil de los autores y los enfoques utilizados. Finalmente, Flores-Mariscal incluye el momento contemporáneo, que inició con el nuevo siglo y se caracteriza por un perfil de investigación compartido por los autores, una visión unificada de la disciplina y la inserción de la literatura nacional en los debates a nivel internacional.

En otro orden de ideas, y por su posición crítica o autorreflexiva, conviene rescatar la metáfora-idea central de Antonio Aguilar Rivera (2009), que el autor ejecuta cuando realiza un balance de la ciencia política en México. En dicha metáfora, en México un bosque se está incendiando y la disciplina es incapaz de entender y explicar cómo y por qué esto ocurre. Con esto, Aguilar Rivera pretende señalar que la ciencia política mexicana debe encontrar respuestas satisfactorias a los problemas del país para no darle espacio a los demagogos de los diferentes espectros ideológicos (Aguilar Rivera, 2009).

En el mismo tenor, se puede cuestionar que la ciencia política mexicana hasta el momento, y siguiendo algunas ideas de José Woldenberg (2015), no ha podido abordar a la democracia y sus problemas, ni mucho menos transmitir una pedagogía de la misma en la sociedad mexicana.

Sin embargo, estas inquietudes escapan a este apartado, donde lo que se pretendió fue tener un marco social 
e histórico nacional de la situación en que está inmersa la ciencia política mexicana, para comprender sus debates. En el siguiente apartado toca dar cuenta del trayecto de la institucionalización de la disciplina en Jalisco.

2. El trayecto de la ciencia política en Jalisco: los programas docentes y la investigación académica

A la pregunta expresa de cuándo surge la ciencia política en Jalisco, y siguiendo a Enrique Suárez-Iñiguez, quien dice que la disciplina nació en nuestro país con la creación de la Facultad de Ciencias Políticas de la UNAM (Suárez-Iñiguez, 2014, pp. 169 y 173), es posible decir que el nacimiento de la ciencia política en Jalisco se dio con la creación de la Facultad de Estudios Políticos, Internacionales y de Gobierno de la Universidad de Guadalajara, en 1989, mientras que la fundación de la Licenciatura en Estudios Políticos y Gobierno, de la misma universidad, en 1991, es el referente para considerar el inicio del proceso de institucionalización de la disciplina en esta región del país (Arellano Ríos, 2010, 2014, 2015 y 2017; Gómez Valle, 2011).

De manera concisa, se puede sintetizar la travesía de la ciencia política en Jalisco en cuatro fases. La primera sería una etapa embrionaria, que abarcaría de 1930 a 1970. En ella, la ciencia política estuvo vinculada con la filosofía política, la historia política, el derecho constitucional y la administración pública como disciplinas.

Después estaría una etapa de formalización e institucionalización, situada en la década de los ochenta cuando, y como fecha significativa, se fundó la ya mencionada Licenciatura en Estudios Políticos y Gobierno, en la Universidad de Guadalajara.

Posteriormente, se situaría una etapa de expansión, durante la década de los noventa y hasta el año 2010, marcada por la creación de diferentes programas de pregrado y 
posgrado en la entidad, esto porque la institucionalización también debe entenderse como un proceso de legitimación y constitución de comunidades epistémicas en un campo profesional en expansión.

Finalmente, desde el año de 2010 hasta la fecha (2018), se ha llevado a cabo un proceso de transición hacia la madurez institucional. ${ }^{7}$ El reto consiste en analizar y estudiar cuáles son los órganos y publicaciones que tiene actualmente la disciplina, el número de instituciones de educación superior, el perfil y la ubicación de sus egresados, los programas diseñados y creados, los ritmos y asimetrías, sus componentes, y qué temáticas atiende la disciplina en la entidad actualmente.

Con mayor nitidez desde mediados de la segunda década del siglo XXI, la ciencia política en Jalisco se está desarrollando en diferentes instituciones de educación superior. Esto hace necesario ver qué programas existen y evaluarlos, lo cual sería una tarea muy ardua pero, siguiendo a Adrián Acosta, lo cierto es que una respuesta acerca de cuál es la concepción de la disciplina se puede encontrar en los programas de estudios a partir de las materias y formaciones que se imparten en ellos. Esto daría una primera distinción sobre si sus concepciones de la disciplina son teóricas o instrumentales, por ejemplo.

También se debe observar en qué instituciones se ofrecen cuáles de dichos programas, así como dónde están sus egresados: "Y mi impresión es que en Jalisco, y sospecho que también en todo México, una vertiente muy potente que va jalando, digamos, a la ciencia política está en su relación con la administración pública" (entrevista personal, 2015c). ${ }^{8}$

7. La idea y denominación emana de la entrevista realizada para este trabajo a Adrián Acosta Silva (entrevista personal, 2015c).

8. En el año de 2016, las licenciaturas en política y gobierno que se ofertaban en Jalisco eran la Licenciatura en Administración Gubernamental y Políticas Públicas Locales y la de Estudios Políticos y Gobierno, ambas en la Universidad de Guadalajara; la Licenciatura en Ciencias Políticas y Gestión Pública, del Instituto 
Lo anterior consecuentemente introduce una concepción pragmática de la disciplina en el pregrado y en el nivel de maestría, esto debido a que sus egresados intentan insertarse en el Gobierno, los partidos, la función pública u otros espacios, y pocos optan por la investigación (entrevista personal, 2015a). De este modo, la Licenciatura en Estudios Políticos de la Universidad de Guadalajara, a veinticinco años de haberse creado, se ha convertido en el semillero de una parte de la clase política, la función pública y la opinión pública local, incluso con proyección nacional en algunos casos (Tabla 1$){ }^{9}$

\section{Tabla 1. Algunos egresados de la Licenciatura en Estudios Políticos y Gobierno (Universidad de Guadalajara) con participación en diferentes espacios públicos (2016-2017)}

Nombre
Clemente Castañeda

Alberto Uribe

\section{Ámbito o cargo}

Exdiputado local y diputado federal.

Coordinador de la fracción parlamentaria de

Movimiento Ciudadano en la LX Legislatura.

Senador de la república a partir de 2018.

Presidente municipal de Tlajomulco de Zúñiga, Jalisco.

Tecnológico y de Estudios Superiores de Occidente (ITESO); la Licenciatura en Administración Pública, en el Instituto de Administración Pública de Jalisco; la Licenciatura en Ciencia Política, en el Instituto Tecnológico y de Estudios Superiores de Monterrey (ITESM), campus Guadalajara; la Licenciatura en Administración Pública, de la Universidad del Valle de México (UVM), campus Guadalajara Sur: la Licenciatura en Ciencia Política y Administración Pública, de la Universidad Autónoma de Guadalajara; la Licenciatura en Ciencia Política y Administración Pública, de la Universidad América Latina (UAL); y la Licenciatura en Ciencias Políticas y Gestión Pública, de la Univer. En el nivel de posgrado, estaban la Maestría en Administración Pública, del Instituto de Administración Pública de Jalisco; la Maestría en Política y Gestión Pública, del ITESO; la Maestría Interinstitucional en Políticas Públicas, de la Universidad de Guadalajara-El Colegio de Jalisco; la Maestría en Administración Pública, de la UVM; y la Maestría y el Doctorado en Ciencia Política, de la Universidad de Guadalajara.

9. La Licenciatura en Estudios Políticos y Gobierno abrió su matrícula en el marco de dos acontecimientos importantes: las elecciones de 1988 y la caída del muro de Berlín (entrevista personal, 20I5b). 


\begin{tabular}{|c|c|}
\hline Nombre & Ámbito o cargo \\
\hline Héctor Insúa & Presidente municipal de Colima, Colima. \\
\hline Cesar Augusto & Presidente municipal de San Gabriel, Jalisco. \\
\hline \multicolumn{2}{|l|}{ Rodríguez } \\
\hline Patricia Vergara & $\begin{array}{l}\text { Consejera electoral del Instituto Electoral y } \\
\text { de Participación Ciudadana (IEPC) de Jalisco y } \\
\text { funcionaria pública. }\end{array}$ \\
\hline Sayani Mozka Estrada & $\begin{array}{l}\text { Consejera electoral del IEPC y funcionaria } \\
\text { pública. }\end{array}$ \\
\hline Mario Ramos González & Consejero electoral del IEPC. \\
\hline Moisés Pérez Vega & $\begin{array}{l}\text { Consejero electoral del IEPC y funcionario } \\
\text { electoral. }\end{array}$ \\
\hline Gabriel Torres & $\begin{array}{l}\text { Exvicerrector de la Universidad de Guadalajara } \\
\text { y director del Sistema Universitario de Radio y } \\
\text { Televisión de la misma universidad. Presidente } \\
\text { de la Asociación de Televisiones Educativas y } \\
\text { Culturales Iberoamericanas (ATEI). }\end{array}$ \\
\hline Francisco González & Primer gerente de cabildeo de la Confederación \\
\hline Vallejo & $\begin{array}{l}\text { Patronal de la República Mexicana } \\
\text { (Coparmex), Jalisco, comisionado del Instituto } \\
\text { de Transparencia, Información Pública y } \\
\text { Protección de Datos Personales del Estado de } \\
\text { Jalisco (ITEI), coordinador de la región centro- } \\
\text { occidente de la Conferencia Mexicana para el } \\
\text { Acceso a la Información Pública (COMAIP), y } \\
\text { titular de la Unidad de Transparencia del IEPC. }\end{array}$ \\
\hline Vicente Viveros Reyes & $\begin{array}{l}\text { Comisionado del ITEI. Actualmente jefe de } \\
\text { gabinete en el Ayuntamiento de San Pedro } \\
\text { Tlaquepaque. }\end{array}$ \\
\hline David Rogelio Campos & $\begin{array}{l}\text { Director del Instituto de Información } \\
\text { Estadística y Geográfica del Estado de Jalisco. }\end{array}$ \\
\hline Gilberto Tinajero & $\begin{array}{l}\text { Director del Consejo Económico y Social del } \\
\text { Estado de Jalisco para el Desarrollo y la } \\
\text { Competitividad, y director de Participación } \\
\text { Ciudadana del IEPC. }\end{array}$ \\
\hline Jesús Gómez Valle & $\begin{array}{l}\text { Director de Comunicación Social en el IEPC y } \\
\text { académico de la Universidad de Guadalajara. }\end{array}$ \\
\hline Ramiro Garzón & Director de Informática en el IEPC. \\
\hline Dolores Ruiz Ambriz & $\begin{array}{l}\text { Vocal ejecutiva del Instituto Nacional Electoral } \\
\text { (INE) en el distrito } 10 \text { de la Ciudad de México. }\end{array}$ \\
\hline
\end{tabular}


Nombre

Dora Luz Espinoza

Ernesto Gama Lozano

Roberto Corona Copado

Vladimir Rico

Rodolfo Aceves

Laura Castro

Jorge O. Navarro

Talina Radillo

Alberto Arellano

Carlos Navarrete

Jesús Ibarra

Karla A. Planter

Ismael Aguilera

Joel Díaz

Annel Vázquez

Anderson

José A. Alatorre

Rodrigo Reyes

Carranza

Rigoberto Silva Robles
Ámbito o cargo

Vocal ejecutiva del INE en la Ciudad de México.

Vocal ejecutivo del INE en Tepic, Nayarit.

Asesor en el Instituto Nacional de

Transparencia, Acceso a la Información y

Protección de Datos Personales (IFAI), el IFE, el

INE y las oficinas centrales del Registro Federal de Electores.

Desempeña funciones administrativas en

Tlajomulco de Zúñiga, Jalisco.

Asesor en el Senado y en la Cámara de

Diputados.

Periodista, columnista y conductora de programas radiofónicos.

Periodista, columnista y conductor de programas radiofónicos y televisivos.

Conductora de programas radiofónicos.

Profesor-investigador en El Colegio de Jalisco.

Profesor-investigador de la Universidad de Guadalajara.

Profesor-investigador del ITESO y miembro del

Comité de Participación Social del Sistema

Estatal Anticorrupción en Jalisco.

Académica y funcionaria de la Universidad de Guadalajara. También ha sido comunicadora y conductora en espacios radiofónicos.

Académico de la Universidad de Guadalajara.

Académico de la Universidad de Guadalajara.

Académica de la Universidad de Guadalajara y miembro del Comité de Participación Social del Sistema Estatal Anticorrupción en Jalisco.

Académico de la Universidad de Guadalajara, exconsejero electoral del IEPC y miembro del Comité de Participación Social del Sistema Estatal Anticorrupción en Jalisco.

Titular de la Unidad de Transparencia del Ayuntamiento de Tlaquepaque.

Presidente del Colegio Estatal de Estudios Políticos y Gobierno.

Fuente: elaboración propia. 
Por lo que toca al momento actual, se debe reiterar que la ciencia política en Jalisco en el nivel de pregrado y maestría y doctorado está muy vinculada con la administración pública. Esto genera una tensión entre si a los estudiantes se les forma para ejercer una disciplina o se les prepara para realizar una profesión (Arellano Ríos, 2014).

En este sentido, la ciencia política como disciplina académica es un campo de estudio y rama del conocimiento que tiene como meta generar conocimientos y elaborar teorías, pero su vínculo con la administración pública le plantea también dar respuesta a exigencias laborales. De este modo, el vínculo con la administración pública, guste o no, condiciona que en las instituciones de educación superior se oferte una ciencia política aplicada, generalmente ligada a las políticas públicas (entrevista personal, 2015c; Arellano Ríos, 2014).

Dicho vínculo también ha condicionado, a decir de Adrián Acosta Silva, la existencia de otras dos tensiones en la ciencia política, tanto de México como del mundo. La primera refiere a la ubicación del estatuto teórico de la disciplina, y la segunda a si dicho estatuto se está perdiendo o diluyendo en otras subdisciplinas. "Hay colegas [a los] que les preocupa que la ciencia política pierda identidad, y quieren volver a lo básico [de la disciplina]: fortalecer su identidad y a su campo, diferenciarse de otros, no contaminarse con otros desarrollos o subordinarla" (entrevista personal, 2015c). El debate se ubica ahí: entre una concepción de la disciplina como muy definida o más amplia.

Por otra parte, siguiendo con la institucionalización de la disciplina en el estado, el Doctorado en Ciencia Política de la Universidad de Guadalajara merece una mención especial como el programa que podría posicionar o no a la disciplina desarrollada en Jalisco a nivel nacional. Dicho doctorado y la maestría en la misma disciplina contaban, a finales de 2017, con dos generaciones cada uno. Para dicha 
fecha, el reto del doctorado estaba en permanecer en el PNPC del Conacyt y ser catalogado como en consolidación. ${ }^{10}$

En cuanto al diseño de este programa, Jaime Preciado argumenta que fue antecedido por un diagnóstico nacional y regional, en el cual se observó que los programas disciplinarios en Jalisco se concentraban en la parte profesionalizante, por lo que se diseñaron una maestría y un doctorado encaminados a la investigación y cuyo diseño interno apuntara hacia la ciencia política multi- o transdisciplinar (entrevista personal, 2015a). ${ }^{11}$

Aunque la creación de un programa doctoral en ciencia política resulta importante para catapultar y fortalecer a la disciplina, de manera que se identifiquen y precisen sus características en la investigación académica, esto no quiere decir que antes no se realizara investigación en sus temáti-

10. El origen del Doctorado en Ciencia Política se debe rastrear en el Doctorado en Ciencias Sociales que impartieron la Universidad de Guadalajara y el Centro de Investigaciones y Estudios Superiores en Antropología Social (CIESAS), unidad Occidente, y que era encabezado por Jorge Alonso. El Doctorado en Ciencias Sociales fue el primer programa de este nivel en el estado, y de él se nutrió la segunda más grande comunidad epistémica del país. Alonso (20II) ha dicho que cuando creó el programa de dicho doctorado en todo el estado no existían los doctores suficientes para hacer uno propio, por lo que se eligió su ejecución interinstitucional. Después, la oferta se amplió, hasta que las instituciones creadoras lo pudieron ofrecer por separado. En el proceso se sumarían otras instituciones como el ITESO y El Colegio de Jalisco, que ampliarían la oferta en este nivel. Más tarde, en la misma Universidad de Guadalajara se crearían más programas doctorales, como el de Ciencia Política, Geografía y Ordenamiento Territorial, Humanidades, y Políticas Públicas y Territorio. Para el propio Alonso, hoy "los programas de ciencias sociales empiezan a crear nichos al interior" (entrevista personal, 20I5d).

II. "Quienes participamos en su formulación y en el proyecto, los cuerpos académicos, provenía[mos] más de una tradición, si me permite, más de sociología política, y había ya un engarce, una apuesta interdisciplinaria; sin embargo, [se decidió que] el posgrado se acotara a la parte política y no fuera un posgrado de la tradición en ciencias políticas y sociales" (entrevista personal, 2015a). El debate de una concepción limitada amplia para el doctorado partió de las líneas de especialidad y peculiaridades de los cuerpos académicos de sus formuladores: I) Gobierno y políticas públicas; 2) instituciones, comportamientos y comunicación política; 3) cultura política, sociedad civil, ciudadanía y movimientos sociales; y 4) política internacional, geopolítica y seguridad, como puede verse en su dictamen de creación (documento en poder del autor). 
cas. ${ }^{12}$ En cuanto a este punto, Adrián Acosta ha señalado que otro camino para observar y valorar la travesía de la ciencia política en Jalisco radica en analizar sus organizaciones gremiales, colegios y redes, en concreto sus características y periodicidad de reunión, así como sus publicaciones (libros, revistas, etc.). En el último aspecto, se puede decir que la ciencia política está en las revistas de ciencias sociales, pero no hay una revista específicamente dedicada a la ciencia política en Jalisco (entrevista personal, 2015c). ${ }^{13}$

Por otra parte:

Es notorio y sabido que diversos investigadores en Jalisco [que] han estudiado los cambios políticos, las transiciones políticas, los partidos, los sistemas de partidos, los sistemas electorales, pasaron luego a [estudiar] temas más específicos, de [estudiar el] cambio institucional a [estudiar] temas de gobernabilidad, temas de gestión pública, Gobiernos municipales, [organizaciones no gubernamentales] ONG, participación ciudadana (entrevista personal, 20I5c).

$\mathrm{Al}$ respecto, conviene rescatar la radiografía que Arellano Ríos (2015) realizó de la ciencia política en Jalisco. En su evaluación, el autor encontró que la disciplina practicada

12. Debe valorarse que el posgrado en ciencia política en la Universidad de Guadalajara está en una fase inicial de implementación. Catalogado como de reciente creación, como ya se dijo, debe aún transitar a su calificación como en desarrollo, y posteriormente a consolidado y de competencia internacional. De suceder, esto implicaría un proceso en el que profesores, estudiantes y egresados comenzarían a dar forma a una ciencia política de Jalisco en varios rubros de las agendas y temas de investigación propios de la disciplina, así como en su inserción en las universidades y centros de investigación. Todo esto será de realización posible sólo en el mediano plazo.

13. Cabe precisar que Adrián Acosta ha hecho referencia en su entrevista a revistas académicas que se encuentran dentro de los cánones o parámetros para ser catalogadas como publicaciones científicas en ciencias sociales. No obstante, existen en el estado dos revistas que tienen a la ciencia política como campo disciplinar: Estudios políticos y Gobierno, del Colegio Estatal de Estudios Políticos y Gobierno -colegio de profesionistas que aglutina a egresados del Departamento de Estudios Políticos de la Universidad de Guadalajara-,y Acta republicana, del Centro Universitario de Ciencias Sociales y Humanidades (CUCSH) de la alma mater estatal. 
en el estado es sólida e interdisciplinaria, pues dialoga y se hibrida con otras perspectivas, pero que sus objetos, temáticas y agendas se articulan en seis vertientes de indagación: 1) la electoral, que contiene al $47 \%$ de los trabajos; 2) la que estudia la transición democrática y el cambio político, con una representación del 21\%; 3) la que se aboca al estudio de las élites, la clase política y los partidos, con 9\%; 4) la que analiza las instituciones y el desempeño gubernamental, con $7 \%$; 5) la que estudia los movimientos sociales, con otro 7\%; y 6) la que estudia la cultura política, con 9\% (Gráfica 1).

Gráfica 1. El estudio de la política en Jalisco

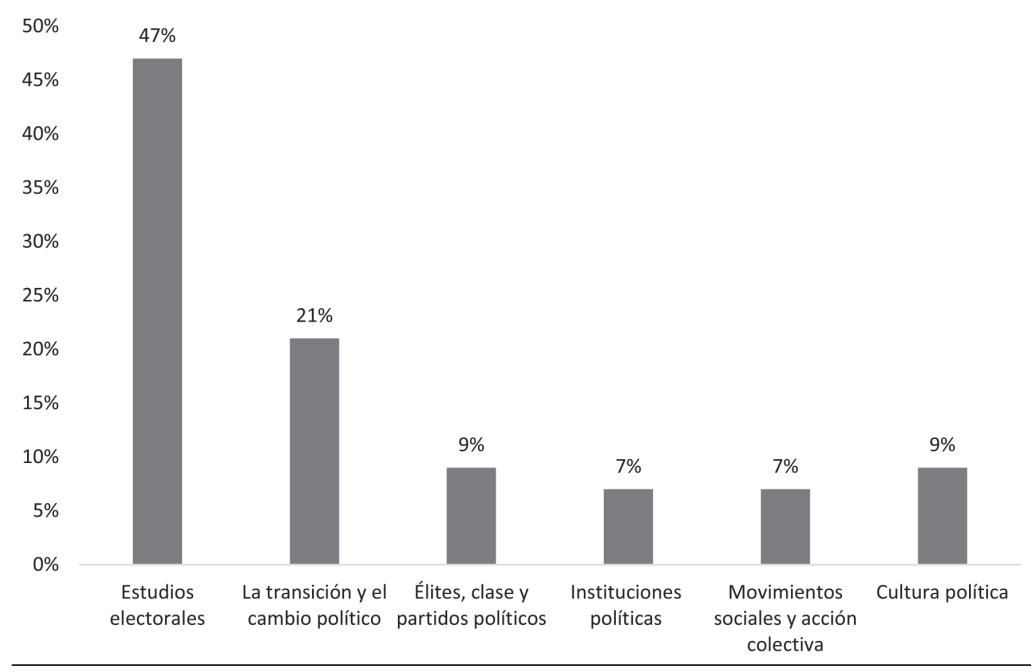

Fuente: Arellano Ríos (2015, p. 160).

En suma, de lo hasta ahora expuesto se puede sostener que hay en el estado conciencia por crear y fortalecer la ciencia política, y al mismo tiempo por hacerla dialogar con otras disciplinas en diferentes niveles. También, y a decir de Jaime Preciado, puede asegurarse que los investigadores locales se están relacionando y tendiendo vínculos con organizaciones académicas muy importantes, como la 
International Political Science Association (IPSA), la Asociación Latinoamericana de Ciencia Política (ALACIP), el Consejo Latinoamericano de Ciencias Sociales (CLACSO), el Consejo Mexicano de Ciencias Sociales (COMECSO) y la Asociación Mexicana de Ciencia Política (AMECIP) (entrevista personal, 2015a).

Por otro lado, tras valorar los procesos de institucionalización de la disciplina y lo que esta produce en el estado, no quedan claras las tendencias teórico-metodológicas que podrían llevar a la ciencia política de Jalisco a contar con una identidad propia y también a valorar sus fortalezas y debilidades. Esto se detalla en el siguiente apartado.

\section{3. ¿Qué tipo de ciencia política debe} constituirse en Jalisco?

En lo hasta ahora expuesto, se ha visto que la ciencia política en Jalisco ha sido cavilada en una visión muy acotada y precisa y otra más amplia. El debate al respecto es actual, pues existen investigadores e instituciones que abogan o defienden los límites, objetos, temas, enfoques o métodos precisos de la disciplina. El presente apartado busca detallar las características de la misma y los problemas que la del estado debería abordar.

Las posiciones en torno a la ciencia política en Jalisco, en un sentido metafórico, se manifiestan y gravitan como un péndulo. La masa suspendida (entiéndase, la ciencia política) y el punto suspendido en un eje horizontal fijo mediante un hilo (sus vínculo y definición y posición entre las ciencias sociales) están entre una perspectiva clásica y otras posiciones que abogan por la inter- y transdisciplinariedad. En otras palabras, entre la posición clásica y acotada de la ciencia política representada por Javier Hurtado, y las concepciones más amplias de transdisciplinariedad, como las de Jaime Preciado y Jorge Alonso, pasando por 
posiciones interdisciplinarias y de mixturas, como la de Adrián Acosta Silva.

Lo destacable es que entre dichas posiciones a la ciencia política en Jalisco se le debería pensar y reflexionar para debatir su definición y precisión. El reto es no dar cuenta de la ciencia política en Jalisco, sino de una ciencia política de Jalisco.

$\mathrm{Al}$ respecto, Javier Hurtado asume una visión de la ciencia política ligada a las cuestiones institucionales. Esta concepción disciplinar está muy cercana al derecho constitucional, la arquitectura y la ingeniería institucional. Hurtado considera, siguiendo a Karl Loewenstein, que "la ciencia política es la ciencia del poder: es la kratología" (entrevista personal, 2015b). Tal definición le permite al académico establecer límites con respecto al estudio de los movimientos sociales, las cuestiones electorales, los asuntos territoriales o geográficos, y las políticas públicas, entre otras temáticas:

[...] para mí, la ciencia política es una disciplina que se encarga de estudiar los procesos a través de los cuales se accede a los cargos gubernamentales, la voluntad popular se transforma en cargos electivos o de autoridad, de representación o de autoridad, y se establecen relaciones entre los detentadores del poder, es decir, entre poderes $y$ órdenes de Gobierno, y a su vez se establecen mecanismos de control sobre el poder (entrevista personal, 2015b).

Cuando el péndulo se mueve hacia posiciones más ampliadas, la postura de Adrián Acosta Silva permite ubicar a la ciencia política en la tradición clásica del estudio del poder, sus actores e instituciones, pero en diálogo con las demás ciencias sociales, lo que podría ofrecer una definición más compleja del poder y la interrelación entre los actores, estructuras y reglas de decisión colectiva (entrevista personal, 2015c), como el mismo académico precisa: 
[...] en realidad, creo que desde los años ochenta, noventa, quizá, en todo el mundo, y en México por supuesto, hay nuevos enfoques que han enriquecido el campo de la ciencia política, es decir, ya no [se ejerce] sólo el estudio del poder con visiones historiográficas, o constitucionalistas, o formalistas, o las tradiciones comparativistas de la política, de tipo Duverger o Sartori, etcétera, sino que en realidad tenemos ahí un conjunto nuevo de temas y campos de la ciencia política que se han derivado hacia [otras] áreas, como las políticas públicas. No [sólo se da] el acercamiento de la ciencia política con la administración pública, con la economía pública, con el derecho público. [Esto] ha dado, digamos, como resultado un marcado interés de ciertas áreas de la ciencia política en el campo de la política, y creo que ahí podríamos tener un área importante, en términos más teóricos, de la irrupción de enfoques como el del neoinstitucionalismo político o neoinstitucionalismo sociológico, que también creo que ha impactado de manera importante la configuración de la ciencia política y, digamos, una serie de derivaciones muy extrañas (entrevista personal, 2015c).

Lo señalado por Acosta Silva ha permitido que la ciencia política se enriquezca, complejice en sus temas y expanda sus fronteras. Para el mismo académico, el desarrollo de la disciplina tiene que ver con los avances de carácter más bien teórico, es decir, con refinar y revisar sus conceptos, con rehabilitar los anteojos con los cuales mira los problemas de poder, y también con incidir en su comportamiento.

En esta doble dimensión se puede vislumbrar el presente y el futuro de la ciencia política:

[...] simplemente porque los problemas públicos sociales no son problemas químicamente puros que puedan ser abordados sólo con las herramientas de una sola disciplina. Me parece que los problemas sociales y públicos, los que tienen que ver con la política y el poder, son problemas poliédricos, es decir, que [se] requiere de varias miradas para tratar, primero, de comprenderlos $y$, segundo, de influir en su 
comportamiento. Me parece que el futuro de la ciencia política va por ahí (entrevista personal, 20I5c).

Por otro lado, el péndulo de las posiciones en la ciencia política también se mueve hacia otras nociones amplias cuando se retoman los planteamientos de Jaime Preciado y Jorge Alonso, quienes representan visiones alternativas y críticas de la disciplina, respectivamente.

Según retoma Jaime Preciado, durante el diseño de los programas de la maestría y el doctorado en ciencia política en la Universidad de Guadalajara, se presentó la discusión disciplinaria y teórica sobre si llamarlos en ciencia o en ciencias políticas. Al respecto, y con la experiencia que los formuladores de los programas tenían en proyectos de posgrado, estos debatían sobre si hablar de una ciencia política era más adecuado para darle centralidad a los programas, aunque sin negar los vínculos de la disciplina con las demás ciencias sociales, o si era necesario hablar de ciencias políticas para hacer alusión a la pluralidad de enfoques en la disciplina (entrevista personal, 2015a).

Al final, se optó por la nomenclatura en singular, y en el diseño se desarrolló una concepción más amplia de la disciplina, muy diferente a la posición interdisciplinar planteada por Adrián Acosta Silva. Al respecto, la posición y definición disciplinar de Jaime Preciado en torno a qué es la ciencia política resulta de interés:

Si bien [la ciencia política] tiene que ver con nuestra convivencia, el manejo de nuestros conflictos, la creación de las instituciones, también se debe considerar una nueva ciencia política, que tiene que ver con la otra política, multidisciplinaria, abierta al pensamiento crítico dentro de la ciencia política, más allá de los debates acotados por la tensión entre lo cuantitativo y lo cualitativo, el cual creemos que es un falso dilema, pues en realidad el pensamiento no tiene fronteras para usar los medios más propicios en la producción del conocimiento,y en ese plano, [también] 
creemos que se debe[n] conciliar lo cuantitativo y lo cualitativo [...], así como no caer en los parroquianismos disciplinarios o de regiones geográficas del mundo (entrevista personal, 2015a). ${ }^{14}$

Si la perspectiva de Preciado acerca de la ciencia política es amplia, la de Jorge Alonso es más radical. Alonso, si bien no considera estar dentro de los debates politológicos, tiene una trayectoria en la investigación en temas políticos y como impulsor de programas en ciencias sociales en Jalisco y México. Estas características de su carrera hicieron necesario considerar su posición al respecto.

Alonso precisa que la investigación debe abrirse a profundas y amplias discusiones, a otras corrientes y visiones, además de que debe ser transdisciplinar, pues para él las disciplinas son cárceles (entrevista personal, 2015d).

Respecto a los debates en la ciencia política, Alonso señala:

[...] creo que tendría, por honestidad intelectual, que decirte que obviamente la ciencia política la hacen los que están metidos en ella, y decir que tiene pies de barro, o que no sirve, o que murió, o lo que sea, yo considero que es un poco bizantino [...]. Muchas personas que están haciendo investigación tienen que ver con la política, y al estar enriqueciendo el conocimiento científico desde las ciencias sociales y

14. Al respecto, habrá que precisar y debatir el concepto de crítica. Se puede entender que Preciado da cuenta de una perspectiva acorde con los movimientos sociales y las propuestas llamadas del sur, que tienen en autores como Boaventura de Sousa Santos, Atilio Boron, Anibal Quijano, entre otros, a académicos que forjan y sientan sus bases teóricas. Desde luego, se trata de una posición y trabajo académicos más militantes y activistas que otras posiciones en el campo disciplinar de la ciencia política. El frente en el que debaten se construye entre quienes ven a la disciplina como con un campo de estudio bien definido y quiénes entienden el pensamiento crítico al emplear el método científico y la falsación, así como un diálogo interdisciplinar en el plano metodológico. Como sea, es innegable que los debates epistemológicos y teórico-metodológicos son también una lucha de poder dentro de un campo disciplinario, en los términos de Pierre Bourdieu. 
este inmenso campo de actividad de la gente, pues se está fortaleciendo y dinamizando la investigación (entrevista personal, 20l5d).

Para el mismo investigador, la problemática tiene que ver con que la política, en todas sus ramas, se está alimentando de una gran cantidad de preguntas, inquietudes, hipótesis de análisis:

Las preguntas y cómo uno se acerca a la realidad [son] lo más importante [...];yo estoy abierto a que los límites de las disciplinas, la sociología, no estén tan delimitados, sino que haya una transdisciplinariedad, es decir, que haya cruces, $y$ [que] los cruces se dan efectivamente por las diferentes caras o perspectivas que se toman ante los diferentes problemas. Se deben mezclar perspectivas cualitativas y cuantitativas, se tienen que mezclar muchas perspectivas. Si te limitas a técnicas, te es más difícil analizar y comprender situaciones tan complejas. Las situaciones políticas regionales y nacionales son muy complejas como para abordarlas con cuestiones lineales o simplificadas (entrevista personal, 20I5d).

Como sea, el futuro de la ciencia política en Jalisco está entre una posición muy clara de volver a temas tradicionales y otra que le implicaría abrirse a nuevas temáticas de manera radical. Siendo así, Javier Hurtado se ubicaría en la primera posición, mientras que los otros entrevistados abrirían la perspectiva disciplinar en diferentes grados y matices. De este modo, existe una posición para la cual la ciencia política es hoy día un campo más amplio, más diverso y sujeto a interacciones con otras ciencias sociales, mientras también continúa vigente la vertiente que Giovanni Sartori planteó hace unos años sobre sus métodos, herramientas y abordajes precisos.

También, se sigue manteniendo la discusión entre visiones más cualitativas de la disciplina y visiones más cuanti- 
tativas (entrevista personal, 2015c). ${ }^{15}$ Incluso, dialogar con las ciencias duras permitiría relativizar y avanzar más allá de la cuestión planteada en lo cuantitativo y lo cualitativo. Por ejemplo, pueden surgir problemáticas políticas en las que se haría necesario dialogar con la teoría del desarrollo, o se precisaría una ciencia política que tomara en cuenta el ambiente geográfico, el contexto, la historia, así como el redescubrimiento de la naturaleza del conflicto entre el Estado y la sociedad (entrevista personal, 2015a).

Al final, la ciencia política es un territorio de tensiones, y eso explica la existencia de varios ritmos y asimetrías en su desarrollo. Si bien se tiene que defender una disciplina con interés en el estudio de los problemas de la política (el poder, el orden político, etc.), también hay que reconocer que estos temas reaparecen en los distintos campos de la acción política, por lo cual hay anteojos de la sociología política, la antropología política, de la economía, los movimientos sociales, por mencionar algunos, que ayudarían al estudio disciplinario. En este sentido, la ciencia política tendrá un futuro importante al interactuar con otras disciplinas (entrevista personal, 2015c).

Las posiciones planteadas anteriormente llevan a cuestionar, retomando de nuevo a Antonio Aguilar Rivera (2009), si la ciencia política está dando respuesta a los grandes problemas del país. En este aspecto, Adrián Acosta Silva dice que para responder a ello habría que tomar en cuenta dos dimensiones: 1) la madurez de la ciencia política como campo disciplinario en México; y 2) su avance institucional importante, es decir, la existencia de un número de escuelas, programas de licenciatura y posgrado, centros, grupos y redes de investigación de la disciplina con los que no se contaba años atrás. La ciencia política en los últimos veinte

15. Para Adrián Acosta Silva, un ejemplo de nuevos campos de estudio está en lo que ha hecho Elster al estudiar la subjetividad política. 
años se ha expandido de manera notable: "Hay una ciencia política que no se tenía hace veinte o veinticinco años, y eso creo que es una buena noticia. Pero, por el otro lado, lo anterior no se ha traducido en su capacidad para resolver o abordar productivamente los problemas, digamos reales, de la política en México" (entrevista personal, 2015c).

Ahora bien, para Acosta Silva esto último, la solución de los grandes problemas del país, quizá no sea uno de los propósitos explícitos de la ciencia política, si bien esta ha avanzado mucho en comprenderlos, con distintos abordajes, escuelas y enfoques, porque es difícil que la comprensión o formación de nuevas generaciones de politólogos traiga soluciones para los problemas duros de México:

[...] creo que ahí tenemos una de las limitaciones de todas las ciencias sociales. Su función fundamental, desde mi punto de vista, sigue siendo [que la ciencia política] como ciencia social está orientada fundamentalmente a la comprensión de los problemas. La resolución de los problemas no [le] corresponde estrictamente, [como] quizás [sí] a alguna de sus afluentes, de sus desarrollos posteriores, que son los que tienen que ver sobre todo con las políticas públicas. Ahí podríamos encontrar mayores desarrollos de la ciencia política [para la solución de problemas], pero ese campo, que cada vez se convierte en un campo [más] autónomo, es el de la política pública (entrevista personal, 20I5c).

La posición de Javier Hurtado a tal interrogante es más sentenciosa. El investigador dice que además de haber una situación de indefinición, se está viviendo una realidad en la cual la ciencia política no alcanza a comprender y procesar los problemas del país. Hurtado sostiene que la disciplina se muestra incapaz, incluso, de interpretarlos adecuadamente por la ausencia de verdaderos politólogos y de liderazgos que llamen la atención a los partidos y la clase política. Para el mismo investigador, la falta de identidad de la ciencia 
política se debe a su mezcla y vínculo con la administración pública (entrevista personal, 2015b). ${ }^{16}$

En otra línea aún más crítica y puntual, Adrián Acosta Silva menciona que, pese a que la disciplina en Jalisco se ha expandido, no hay todavía una institución de ciencia política en el estado que genere escuela y politólogos y que sea un referente a nivel nacional:

Creo que aquí hay una diferencia importante. No estoy tan seguro, [pero] efectivamente no hay encuestas o enfoques que diferencien $y$ distingan a Jalisco de lo que hacen las otras escuelas de ciencias políticas del país, o sea, la Facultad de Ciencias Políticas de la UNAM sigue siendo una referencia muy importante, $y$ en algunas [otras] instituciones se han desarrollado posgrados, maestrías o doctorados, en ciencia política, y son referentes, pero la Universidad de Guadalajara, u otra institución de educación superior de Jalisco, no parecen tener el carácter de reconocimiento nacional (entrevista persona, 2015c). ${ }^{17}$

16. Para Javier Hurtado, quienes han asumido la inquietud de abordar los problemas del país en la vertiente de la reforma del Estado han sido los académicos del Instituto de Investigaciones Jurídicas de la UNAM: "yo no sé cómo le hacen, pero esos ni son abogados ni son politólogos, y sin embargo hablan con mayor propiedad de los fenómenos del poder político, de la configuración, que muchos que se dicen politólogos, que son más bien sociólogos o antropólogos. Ese es el ideal, a mí me parece, con más ingredientes de ciencia política. Yo le pondría de lo que yo esperaría de un politólogo, eso es lo que se requiere, no electoralistas, no estudiosos de los movimientos sociales. Yo creo que lo que el país requiere es un perfil como el de los investigadores del Instituto de Investigaciones Jurídicas" (entrevista personal, 20I5b).

17. El debate al respecto sigue abierto. En el ámbito nacional, se mantiene una fragmentación interna en la ciencia política, una parte de la cual todavía no se asume como una ciencia, en singular, y se sigue pensando en plural (ciencias políticas). Empero, no hay duda que la democratización del país la ha impactado favorablemente en su crecimiento (Barrientos del Monte, 2015). 


\section{Conclusiones}

Se puede sostener que la ciencia política de Jalisco no está ajena a las discusiones politológicas nacionales e internacionales y de las ciencias sociales en general. En el balance que se puede hacer de su trayecto, su institucionalización y su quehacer académico hay asimetrías: 1) en el nivel de pregrado hay consolidación y diversificación; 2) en el posgrado hay un progreso hacia la institucionalización; 3) no termina de insertarse en la discusión y reflexión de los problemas de la entidad federativa en que se hace, ni del país en general; y 4) el debate y su inserción en él se dan de forma individualizada, y la comunidad, si bien sitúa las grandes coordenadas de reflexión teórico-metodológica de largo aliento, deja el esfuerzo hasta ahí. Por lo tanto, la valoración que se puede hacer de la ciencia política en Jalisco es que esta no sólo se encuentra dispersa y fragmentada, sino que no tiene una identidad propia.

Ya en una reflexión puntual, en el campo académico local, las concepciones del estado de la disciplina parten de una visión acotada y llegan hasta otras que son amplias. Se avanza de una concepción de la ciencia política vinculada a la cuestión institucional del Estado, el sistema y el régimen político, hacia perspectivas más críticas y que abogan por la transdisciplinariedad, pasando por la interdisciplinariedad o la hibridación.

Si bien la ciencia política en Jalisco nació formalmente con la creación de la Licenciatura en Estudios Políticos y Gobierno, en la Universidad de Guadalajara, ha transitado por cuatro fases: una embrionaria, otra de formalización, una de expansión y otra de madurez institucional. Lo anterior, se reitera, permitió que la disciplina en el nivel de pregrado tuviera mayor fortaleza institucional, y que en el posgrado iniciara su etapa de desarrollo institucional. 
La fortaleza de la ciencia política en Jalisco en el pregrado explica, mayoritariamente desde la universidad pública, que este sea el espacio de construcción de redes políticas y profesionales para el reclutamiento e inserción de politólogos en dos campos sociales principalmente: 1) la política y la función pública; y 2) la opinión pública y los medios de comunicación. Aunque al respecto hacen falta estudios más detallados, parece posible decir que el pregrado cumplió su objetivo e intención central al respecto. Ahora bien, habrá que esperar para saber cómo incide el posgrado en la investigación, la generación de conocimiento y los debates de mayor aliento de la disciplina en el estado.

Por lo que toca al campo de la investigación en temas políticos y públicos, se puede constatar que la ciencia política, o la investigación en temas políticos, si se quiere precisar, tiene solidez, la cual se refleja en el estudio de los temas electorales, el cambio político y las políticas públicas, aunque falta que se indague en otras temáticas. Lo más sustantivo es que se amplíe y profundice la reflexión teórica. Al final, la sentencia más fuerte, que se convierte en valoración categórica, es que pese a los esfuerzos por fortalecer la ciencia política en Jalisco, aún no hay una institución, centro de investigación o comunidad epistémica que la proyecte a nivel nacional y le proporcione una identidad y rasgos propios. El reto es que la discusión y el trabajo colegiado obliguen a los miembros de la comunidad científica a una base mínima donde coincidan y formen una escuela politológica con proyección nacional. ¿Cuáles son o serán esos elementos básicos? La pregunta está abierta.

Acosta Silva, A. (2009). “La política en México: ideas, anteojos y cristales”. Andamios, 6(I I), I0 I-I 28. Recuperado de:https://andamios.uacm.edu.mx/index.php/andamios/ article/view/I 54 
Bibliografía
Aguilar Rivera, J.A. (2009).“"El enclave y el incendio”. Nexos. Recuperado de: http://www.nexos.com.mx/?P=leerarticulo\&Article $=12$

Alarcón Olguín, V. (coord.) (2006). Metodologías para el análisis político. Enfoques, procesos e instituciones. México: UAM y Plaza y Valdés.

Alarcón Olguín, V. (20I2). "La ciencia política mexicana. Reflexiones sobre su pasado, presente y porvenir”. Politica. Revista de ciencia política, 50(I), 3 I-57. Recuperado de:https://revistapolitica.uchile.cl/index.php/RP/article/ download/22648/23968/

Alonso, J. (20I I). “Introducción”, en J. Alonso (ed.), Veinte años del Doctorado en Ciencias Sociales del CIESAS-Occidente (más de cien voces) (Pp. 15-22). México: CIESAS.

Arellano,A. (24 de marzo de 20I5a). Entrevista realizada a Jaime Preciado Coronado, profesor-investigador de la Universidad de Guadalajara. Guadalajara, México.

Arellano, A. (25 de marzo de 20I5b). Entrevista realizada a Javier Hurtado González, profesor-investigador de El Colegio de Jalisco. Guadalajara, México.

Arellano, A. (16 de abril de 2015c). Entrevista realizada a Adrián Acosta Silva, profesor-investigador de la Universidad de Guadalajara. Guadalajara, México.

Arellano, A. (29 de mayo de 20I5d). Entrevista realizada a Jorge Alonso Sánchez, profesor-investigador del Centro de Investigaciones y Estudios Superiores en Antropología Social (CIESAS), unidad Occidente. Guadalajara, México.

Arellano Ríos, A. (20I0). "La gestión pública: un nuevo enfoque para los viejos problemas burocráticos”, en A. Arellano Ríos, N. García Vázquez, R.Arias de la Mora, y E. Rodríguez Pérez (eds.), Del discurso a la práctica. Cuatro estudios acerca de la administración pública en Jalisco (pp. 29-63, I63-172). Zapopan: El Colegio de Jalisco. 
Arellano Ríos, A. (20I3). "El estudio de la política. Rutas, temas, enfoques y perspectivas", en A. Arellano Ríos, y N. García Vázquez (coords.), Ciencia política y políticas públicas en Jalisco.Teoría y metodología (pp. 19-53). Zapopan: El Colegio de Jalisco.

Arellano Ríos, A. (20|4). "Los programas educativos en administración pública en Jalisco:tendencias y agenda de trabajo". Acta republicana. Política y sociedad, I3(I3), 5 I-59. Recuperado de: http://148.202.18.157/sitios/publicacionesite/pperiod/republicana/pdf/ActaRep /3/5.pdf

Arellano Ríos,A. (20I5). “La ciencia política en el estado de Jalisco: un balance de su institucionalización”. Estudios políticos, (34), I39-165. doi: http://dx.doi.org// 0.1016/j. espol.2015.05.002

Arellano Ríos, A. (2017). "La ciencia política: reflexiones desde el ámbito subnacional”, en F. Barrientos (ed.), Historia y balance de la ciencia política en México (pp. 209-234). México: Tirant lo Blach y Universidad de Guanajuato.

Aziz Nassif, A. (1999). "La ciencia política: empirismo, fortaleza vacía, hibridación y fragmentos”, en P. González (coord.), Ciencias sociales: algunos conceptos básicos (PP. 67-87). México: Siglo XXI y CEIICH-UNAM.

Barrientos del Monte, F. (20/3). "La ciencia política en América Latina. Una breve introducción histórica”. Convergencia. Revista de ciencias sociales, 20(6I), I05-I 33. Recuperado de:https://convergencia.uaemex.mx/article/ view/ I052/78 I

Barrientos del Monte, F. (20I4). Buscando una identidad. Breve historia de la ciencia política en América Latina. México: Fontamara y Universidad de Guanajuato.

Barrientos del Monte, F. (20I5). "Crecimiento e institucionalización de la ciencia política en México”. Revista de ciencia política, 35(I), 95-I20. Recuperado de: http:// www.redalyc.org/pdf/324/324393 I 9006.pdf 
Bibliografía
Barrientos del Monte, F. (ed.) (20I7). Historia y balance de la ciencia política en México. México: Tirant lo Blach y Universidad de Guanajuato.

Bulcourf, P., Gutiérrez, E., y Cardozo, N. (20I5). "Historia y desarrollo de la ciencia política en América Latina: reflexiones sobre la constitución del campo de estudios". Revista de ciencia política, 35(I), I79-199. Recuperado de: http://www.redalyc.org/pdf/324/324393 1 9009.pdf

Díaz-Bravo, L.,Torruco-García, U., Martínez-Hernández, M., y Varela-Ruiz, M. (20I3). “La entrevista, recurso flexible y dinámico”. Investigación en educación médica, 2(7), I62-167. Recuperado de: http://riem.facmed.unam.mx/ node/47

Flores-Mariscal, J. (2016). “Evolución de la literatura sobre el estado de la ciencia política en México (1947-20I5). Otra mirada del proceso de desarrollo de la disciplina". Revista mexicana de ciencias políticas y sociales, LXI(227), 55- 102. Recuperado de: http://www.revistas.unam.mx/ index.php/rmcpys/article/view/50526/49066

Gómez Valle, J. (20I I). “El estudio de la ciencia política en Jalisco. La creación de la Facultad de Estudios Políticos, Internacionales y de Gobierno de la Universidad de Guadalajara”. Estudios sociales, (8), 227-239.

Loaeza, S. (2005). "La ciencia política: el pulso del cambio mexicano". Revista de ciencia política, 25(I), 192-203. Recuperado de: https://repositorio.uc.cl/bitstream/ handle/ I I 534/ I I 099/000382498.pdf? sequence = I \&isAllowed=y

Reveles Vázquez, F. (coord.) (20I2). La ciencia política en México hoy: ¿qué sabemos? México: Plaza y Valdez y FCPYS-UNAM.

Rivera, M., y Salazar-Elena, R. (20I I). "El estado de la ciencia política en México. Un retrato empírico". Política y gobierno, XVIII(I), 73-I08. Recuperado de: http:// 
www.politicaygobierno.cide.edu/index.php/pyg/article/ view/ /68/85

Sánchez Noriega Armengol, M. (2015). "Panorama de las revistas sobre ciencia política en Iberoamérica". Estudios políticos, (36), I I-33. Recuperado de: http://www.scielo. org.mx/pdf/ep/n36/n36al.pdf

Sartori, G. (2004). “iHacia dónde va la política?”. Política y gobierno, XI(2), 349-354. Recuperado de: http://www. politicaygobierno.cide.edu/index.php/pyg/article/ view/330/240

Suárez-Iñiguez, E. (20 I4).“El largo camino hacia la autonomía y la institucionalización de la ciencia política, en México y en el mundo". Estudios políticos, (3I), I65-183. Recuperado de: http://www.revistas.unam.mx/index.php/rep/ article/view/44II8/39910

Vidal de la Rosa, G. (20I3). Ensayos sobre la ciencia política en México y Latinoamérica. México: UAM Azcapotzalco.

Woldenberg, J. (20I5). La democracia como problema (un ensayo). México: El Colegio de México y UNAM.

Bibliografía 